\title{
SUMMARY AND REVIEW: INTERACTION OF OBSERVATIONS AND THEORY OF STELLAR INTERIORS
}

\author{
STEVEN D. KAWALER \\ Department of Physics and Astronomy \\ Iowa State University, Ames, IA 50011 USA
}

"The star is never wrong." - Leslie Kawaler, to Ed Nather

\section{Introduction: Observational Approaches to Studying Stellar Interiors}

As we move into the next century of stellar astronomy, it is helpful to consider the modes by which stellar astronomy has advanced in the modern era. At this conference, our attention has been focussed on precision measurement of stellar fundamental properties such as mass, luminosity, and radius. This mode of inquiry is one of several that have borne fruit. In this review, I will discuss some of the topics that have arisen here with respect to how our understanding of stellar interiors progresses through interaction between theory and observation.

At some level, stars are relatively simple. However, sufficiently detailed study of individual stars, in particular our Sun, reveals they can be extremely complex. One of the issues that we constantly struggle with is in defining which observed properties are important to understand and which properties might be simply idiosyncratic. Study of unusual, prominent, or pathological stars sometimes proves to be meaningful for studies of more average subjects, while at other times these prominent stars can be misleading. Within this category fall some "famous" stars such as Mira, Betelgeuse, and Sirius. Here too we find objects whose fame is rooted in historical as well as in modern studies: the Crab pulsar, SN 1987A, and so on. Lesser known stars have served as the prototypes for important classes of objects, including (again) Mira, $\beta$ Pictoris, and PG 1159-035.

In contrast to the prominent examples, there are several prototypical objects that are unremarkable save for their proximity to Earth and the ease with which we can study them. The principal example is of course our Sun; an otherwise (we hope) ordinary G2V star that we can study in 
incredible detail. It is justifiably the star where all stellar interior theory begins... if you can't get the Sun right, then don't bother with globular cluster isochrones!

Beyond prominent or average "individuals", focus groups are also important areas of study. Because of the long time scales over which star change the ensemble properties of samples of stars are needed to learn about stellar evolution. The importance of star clusters for understanding stellar evolution is clear; examples of clusters that have been, and will continue to be, key objects include 47 Tuc, $\Omega$ Cen, and the Hyades. These objects have grown in importance over the years because understanding them in the greatest possible detail reveals important new insights into the physics of all stars. For example, matching the morphology of cluster color-magnitude diagrams has led to the discovery of the likely effects of convective overshoot in stellar cores (as reviewed by Cesare Ciosi) and continual progress in determining stellar ages (as reviewed by Don Vandenberg).

Additional important constraints come from more extensive samples, such as all-sky surveys, luminosity functions, and so on. The collection of all white dwarf stars in our part of the Galaxy, for example, provides important data on the star formation history of our neighborhood. As discussed by Gilles Chabrier at this meeting, the white dwarf luminosity function also plays an important role in stimulating studies of the physics of white dwarf interiors - including the process of crystallization and phase separation in degenerate matter. The collective properties of the variable stars uncovered by the MACHO and OGLE surveys are extremely illuminating for the understanding of stellar pulsation, as illustrated by Kem Cook and Dante Minniti at this meeting.

\section{2. "Fundamental" Properties}

Fundamental observed properties of stars have always played crucial roles in theoretical investigation of stellar structure and evolution. As is evident from the discussions at this meeting, they will continue to provide technical challenges, the solutions of which will push the envelope of technology. As a faculty member in a department of Physics and Astronomy, I've recently been exposed to heavy ion collider physics through our nuclear physics group. They are part of the RHIC (Relative Heavy Ion Collider) experiment at Brookhaven National Laboratory. One of the techniques that RHIC is employing to study the head-on collision of accelerated gold nuclei is, of all things, Hanbury-Brown Twiss interferometry for localization of interaction products in the search for the quark-gluon plasma. I expect that continued efforts in fundamental stellar astronomy will continue to develop techniques with broad application. 
The revolution in stellar astronomy truly began with the first determination of stellar distances in the middle of last century. With distances known, the energy output of the stars beyond the Sun could be determined, and stellar masses calibrated. The assembly of catalogs of stellar distances enabled the "invention" of the modern $\mathrm{H}-\mathrm{R}$ diagram, providing systematic relations amongst the fundamental stellar parameters for theoretical investigation. Rapid progress included the mass-luminosity relation, and its explanation on theoretical grounds, and quantitative exploration of the direction and rate of stellar evolution.

This is a continuing history, as made apparent during this meeting. Calibration of the cosmic distance scale relies upon the first steps in the chain. Precise masses and luminosities of Cepheid variables, RR Lyra and other horizontal branch stars are universal concern. The imminent release of the Hipparcos results, as previewed here by C. Turon, is eagerly anticipated. Progress in development of CCD astrometry is allowing ground-based parallaxes of faint (and relatively distant) stars (see the review in this volume by C. Dahn) that we could not have hoped for a generation ago.

Beyond mass and luminosity, what other "fundamental properties" do stars possess? Principal competition for another quantity is between some measure of the temperature, and some measure of the radius. Both temperature and radius demand further qualification. By radius does one mean the position of last scattering? If so, at what wavelength? If defined as where the density drops to zero, how does one measure such a thing? Similar ambiguities confront the use of temperature as a fundamental quantity. Is "effective temperature," as determined via the Stefan-Boltzmann law, sufficiently fundamental? Even if so, how does one measure such a quantity? If the star is not perfectly spherical, what then? As illustrated in Bob Kurucz's review, stars have temperature gradients along their surfaces caused by convective motions, and active regions. Spatial temperature variations also change with time.

One might argue that a truer "fundamental quantity" is stellar surface area! After all, it is the surface area that links the measured fluxes with stellar luminosity, independent (to high order in most round-ish stars) of star shape. That is, of course, if one knows the distance to the stars (or their angular diameters).

Stellar evolution theorists typically discuss models of given masses, luminosities and effective temperatures. But comparison with observations requires transformations of these ideal quantities into "observables" such as colors and broadband magnitudes. The difficulties of these transformations, described by many at this meeting, remain with us. It is rare that determination of $T_{\text {eff }}$ to $1 \%$ or less is of great importance within this climate. However, for some stars the determination of $T_{\text {eff }}$ to $10 \%$ or more remains 
a challenge to the observers. It is in these difficult cases that progress in the study of stellar interiors depends on the observational constraints on these fundamental quantities.

As a relatively recent example, in 1987 the temperatures of hot white dwarfs such as PG 1159-035 were very poorly known. But with the work of Werner and Dreizler on NLTE atmospheric models in the late 1980s and early 1990 s, these uncertainties have been reduced to well below $10 \%$. With this advance, seismological studies of these stars, described in the next section, became possible. In the future, more accurate determination of the fundamental parameters of a wide variety of pulsating stars, including the pulsating sdB stars described above, the rapidly oscillating Ap stars, and $\delta$ Scuti stars will provide a key to unlocking their seismic secrets.

Another fundamental property of stars is angular momentum. All stars rotate. Observationally, the amount of rotation varies significantly between otherwise identical stars. The influence of rotation ranges from mechanical (through partial hydrostatic support in rapidly rotating stars as well as rotationally-induced mass loss) to thermal (through horizontal heat transport in circulating material) and compositional (via rotationally driven mixing in stellar interiors).

At this meeting, we heard about several of the effects of stellar rotation. Contrary to what many of us learned in being introduced to stellar interiors, the effects of stellar rotation are frequently of 0 th order, not first or second. Certainly during star formation and pre-main sequence evolution, angular momentum and its redistribution (and loss) is a controlling factor. Coupled to the angular momentum problem is the issue of mass loss, which, as demonstrated here by Andre Maeder's discussion, clouds the whole issue of the main sequence for massive stars. During main sequence evolution and beyond, rotation significantly alters the evolutionary tracks of stars, as shown in N. Langer's review.

Redistribution of angular momentum must occur as a consequence of the onset of rotational instabilities in regions of steep angular velocity gradients. Such redistribution can result in the redistribution of material. This, in turn, causes mixing of composition, and provides observational signatures as "peculiar" surface abundances in stars. The process of rotational mixing and composition transport was discussed by a variety of people at this meeting, including Andre Maeder, Corinne Charbonnel, and Charles Proffitt.

\section{A Case Study: Stellar Seismology}

The brightness of stars at various colors is not necessarily constant with time. Classical variable stars have long been evidence of this, but new 
techniques are showing that many stars are multiperiodic variables at a very small level, both photometrically and spectroscopically. These subtle variations are the signal for asteroseismology, which has the potential to revolutionize the study of stellar interiors. Asteroseismology involves long observing runs using large telescopes to look at relatively bright stars. Recent success in this area is very promising. Here, I briefly mention a few areas of progress in asteroseismology. There are others, including the newlydiscovered multiperiodic sdB stars, the rapidly oscillating Ap stars, $\delta$ Scuti stars, etc.

Jørgen Christensen-Dalsgaard reviewed some of the progress in helioseismology in this meeting, and due to lack of time could only provide a few illustrative examples. One remarkable result is of broad impact in physics and astrophysics. The measured solar oscillation frequencies determine, through inversion, the sound speed in the solar interior. Comparison with the current "best" solar model shows agreement to remarkable precision much better than $1 \%$ everywhere within the model. The independentlyconstructed solar model to which this inversion is compared includes the most accurately known input physics (equation-of-state, opacities, nuclear reaction cross-sections). This result is the essence of interaction between observations and theory. As he notes, this places stringent constraints on solutions of the solar neutrino problem, placing the burden firmly on particle physicists.

Other helioseismological results made possible by ground based (GONG, BISON, LOWL, etc.) and space-based (SOHO) observations include measurement of the solar rotation as a function of depth and latitude, the subsurface structure in the vicinity of sunspots and other active regions, mapping of large scale convection, etc. All of these results challenge current theoretical pictures of the Sun. By extension, our view of stellar interiors and evolution will never be the same.

Helioseismic results describe a single star at a single time in its history. Modifications of our understanding of the equation-of-state of stellar matter are appropriate for the line described by the Sun in the $(\rho, T, \mu)$ volume of phase space; we all know what fraction of a volume is covered by a line. Study of stars in a comparable level of detail would certainly broaden our understanding of all aspects of stellar physics.

Compared to the stars, the Sun is "easy pickings" for seismological study. We get many more photons from the Sun, and we can resolve its surface. This allows high signal-to-noise observations that reveal very small amplitude variations, and spatial modulation allows unambiguous mode identification. To even detect solar-like oscillations on solar-type stars, a combination of cleverness, benevolent telescope allocation committees, and a degree of luck (beyond clear skies) is needed. 
Hans Kjeldsen gave compelling justification for undertaking the challenge to detect and measure these modes. Since convection plays a central role as a mechanical stimulator of the oscillation modes, a wide area of the $\mathrm{H}-\mathrm{R}$ diagram may be populated by multimode nonradial pulsators analogous to our Sun. Kjeldsen and his collaborators, and many other groups, have attempted to detect oscillations of solar-type stars. As he reviewed at this meeting, this is indeed a difficult observational problem. Possible detection of oscillations in $\eta$ Bootes await confirmation (Brown et al. 1997). Kjeldsen's progress report on $\alpha$ Cen is tantalizing, and illustrates again the technical challenges of these important observations.

Probably the most successful investigations in asteroseismology involve the pulsating white dwarf stars. Seismological analysis of these stars allows unprecedented access to their interiors. The pulsation modes in these stars, nonradial $g$-modes, provide a sensitive indicator of the mass of the star and can also be used to determine depth below the surface where steep composition gradients occur (Kawaler \& Bradley 1994), among other things. Papers from recent ground based studies (for example, the papers by the Whole Earth Telescope collaboration) describe this procedure in some detail.

Observational results from white dwarf seismology pose a significant challenges to theoretical models. In one example, the thickness of the heliumrich surface layer of the hottest white dwarfs (Kawaler \& Bradley 1994) was measured to be three orders of magnitude larger than that in cooler pulsating helium-rich white dwarfs (Bradley \& Winget 1994). This challenged the notion that they were two links of the same evolutionary change until Dehner (1996, see also Dehner \& Kawaler 1995) showed that diffusive purification of the surface helium layer produced a composition transition zone that reached the correct position at the time the models reached the cooler pulsational instability.

Interaction between observation and theory, and between different modes of observation, is an important facet in white dwarf pulsations. Spectroscopic determinations of the abundances, effective temperatures, and gravities (as reviewed by Dreizler at this meeting) provide essential constraints on seismological models (i.e. O'Brien et al. 1996, Kawaler et al. 1995), while independent determination of these quantities using both techniques foster confidence in both methods (Kawaler \& Bradley 1994).

An additional benefit of seismological studies of white dwarf stars is that they can yield distance determinations. Asteroseismic distances to two stars have been independently confirmed. Bradley and Winget (1994) published an asteroseismic distance for the DB star GD358 that matched parallax measurements, and Kawaler et al. (1995) determined the distance to the PG1159 star PG2131 that was confirmed via the spectroscopic parallax of its K-dwarf companion by Howard Bond (1995, private communication). 


\section{The Road Ahead... AGB Thermal Pulse Dynamics as a Se- lected Example}

As discussed by several at this conference (i.e. Lattanzio, Boothroyd, Charbonnel) the chemical abundances in AGB stars, and indeed the carbon-star phenomenon, is not easily solved with standard hydrostatic evolutionary models. Perhaps an understanding of the dynamical aspects of helium shell flashes in AGB stars will allow solution of this classic problem?

One of the fundamentals that we learn in the early days of stellar evolution courses is that there is a "hierarchy of time scales" that is usually obeyed within stars. That is, $\tau_{d y n} \ll \tau_{\text {therm }}<\tau_{n u c}$ during most phases of stellar evolution. It is this hierarchy that allows us to compute the evolution of stars as a quasi-static process. With the thermal and dynamical time scales shorter than the time scale for nuclear transformations, we can "freeze" the composition of the stellar material to follow thermodynamic transformations generated by a composition change. Similarly, if the time scale for heat flow is long compared to that for dynamical (hydrodynamical) adjustments, then we can assume hydrostatic equilibrium is maintained for the purposes of thermodynamic adjustments to a changing energy flux. By taking time steps that are comparable to the thermal time scale, the assumption of hydrostatic equilibrium is accurate to high accuracy. Th time step is sufficiently short that nuclear processing results in only small changes to the abundance of relevant elements over the time step.

If, however, the time scale for dynamic adjustments is comparable to the time scale for heat flow, then these assumptions are no longer valid. The equations solved by a standard hydrostatic stellar evolution code do not describe the stellar material in this circumstance. Such a standard stellar evolution code may in fact produce models that look nice and converge quickly, but such models are solutions to equations that are not relevant to the real situation that they are supposed to be modeling. Similarly, if nuclear transformation occur at a rate that is fast (i.e. comparable to the time scale for heat flow) the hydrostatic code will produce irrelevant results.

In thermally pulsing AGB stars, the worst of all situations can occur. During a thermal pulse, the helium-burning shell drives a convective region. In the convective region, the thermal and dynamical time scales are comparable. But this convective region rides atop the nuclear burning shell, which is experiencing a mild runaway. The coupling results in all three time scales being comparable in the shell-burning region.

To fully resolve the temporal behavior of the star during the pulse, and to "accurately" track nucleosynthesis through the pulse, time steps are required to be extremely short ... years or less. However, the dynamical time scale for these stars is of this order. Therefore, the evolutionary calculations are implicitly demanding hydrostatic evolution when, in fact the envelope 
of a real AGB star is free to respond on the same time scales in a dynamic way. Also, within the burning region, the luminosity can be enormous, and work out to be larger than the (local) Eddington luminosity. Under such conditions, the material there is dynamically unstable.

Clearly, we are making mistakes when we model the evolution of AGB stars. Perhaps when these mistakes are corrected, the observed abundances of AGB stars will be less mysterious.

\section{Conclusions}

The theoretical study of stellar interiors continues to rely on observational constraints, as it did at the turn of the 20th century and earlier. However, this reliance has now bifurcated. On the one hand, more detailed observations are pushing hard on the theoretical assumptions of spherical symmetry, global thermal and hydrostatic equilibrium, lack of importance of rotation and magnetic fields, etc. As observers pin down the abundances and fundamental properties of stars, theorists will have to work hard to produce realistic models that explain these observations in all of their detail.

On the other hand, there remain significant regions of the $\mathrm{H}-\mathrm{R}$ diagram where parameters of the stars are not precisely known. These include, on the cool side, the formation and evolution of very low-mass stars, the pulsation/convection/mass loss interaction in AGB stars, and the production of planetary nebulae. On the hot side, the evolution of massive stars with rotation and mass loss remains an exciting and controversial research area.

In the early days of helioseismology, an important result was the measurement of the depth of the solar convection zone. While "modern" helioseismology has provided a large number of other important discoveries, such a measurement of the convection zone boundaries in other stars would profoundly affect our understanding of stellar interiors. To do so requires effort as a community to pursue asteroseismological observations. The only way for this to happen is for the stellar astronomers to educate themselves and the broader astronomical community about the potential benefits. Only then will telescope allocation committees and others facilitate the development of a new observational paradigm for stellar astronomy.

All this, and we barely discussed the generation, modulation, and physical effects of stellar magnetic fields! There is much to be done for observers and theorists alike.

\section{Acknowledgements}

The author gratefully acknowledges support from the Organizing Committees, NSF Young Investigator Award AST-9257049, and the NASA Astro- 
physics Theory Program.

\section{References}

Bradley, P. \& Winget,D. (1994), Ap. J., 430, 850.

Brown, T., Kennelly, E., Korzennik, G., Nisenson, P., Noyes, R., \& Horner, S. (1997), Ap. J., 475, 322 .

Dehner, B.T. (1996), Ph.D. Dissertation, Iowa State University.

Dehner, B.T. \& Kawaler, S.D. (1995), Ap. J. Lett, 445, L141.

Kawaler, S.D. \& Bradley, P.A. (1994), Ap. J., 427, 415.

Kawaler, S.D. et al. (the WET collaboration) (1995), Ap. J., 450, 350.

O'Brien, M.S., Clemens, J.C., Kawaler, S.D., \& Dehner, B.T. (1996), Ap. J., 467, 397.

\section{DISCUSSION}

ARNOLD BOOTHROYD: Rotation is also important on the RGB (see, e.g., Charbonnel) to provide a mixing mechanism for the observed abundance anomalies. When a star becomes a giant, the core contracts and the envelope expands. There will be shear at the core boundary and we expect mixing. This may also have an effect when an HB star becomes and AGB star; rotational mixing may also affect He-shell flashes (thermal pulses). Since $\tau_{\text {nuc }} \sim \tau_{\text {conv }} \sim \tau_{\text {dyn }}$ in the He shell flash, it would not be surprising if effects of non-sphericity were significant.

ANDRE MAEDER: I agree that the treatment of shears is essential. In particular, shears and steep $\mu$-gradients occur at the same place and thus any mixing in a region with a steep $\mu$-gradient would considerably affect the evolution.

NORBERT LANGER: I agree that the mixing of elements and angular momentum through the strong $\mu$ - and entropy gradients produced by nuclear shell sources is a fundamental problem. There may be a simple way to check this, i.e., to compare the specific angular momenta of white dwarfs and main-sequence stars. This would tell us how much angular momentum gets sucked out of the core into the envelope during the post-main-sequence evolution.

ROBERT KURUCZ: In your statement about getting observing time for asteroseismology you show a misapprehension of our basic knowledge. We do not know the abundances in the Sun to $10 \%$. In other stars it is worse. The opacities that go into seismology models depend on these abundances. One has to get high- resolution, high-signal-to-noise spectra to fix the stellar parameters. It takes a long time to make the observations and a long time to analyze them.

STEVE KAWALER: It depends on what one is after. Seismology can allow us to determine the positions of convective zone boundaries (core and envelope); we have only theory right now. To me, it is much more exciting to measure, for example, the convective-zone mass in Procyon than to know better the solar nickel abundance. We 
can do so much with seismology, even allowing for uncertainty in composition, that not to do so would be a shame.

TIM BEDDING (also responding to Kurucz): In open clusters you can assume (perhaps wrongly) that all stars have the same metallicity.

JゆRGEN CHRISTENSEN-DALSGAARD: In fact, we probably all agree. For asteroseismic analysis is it crucially important to have as accurate information as possible on the 'classical' stellar parameters, such as mass, luminosity, effective temperature and composition, from traditional observations. Only with such information is it truly possible to use the oscillation frequencies to probe details of the deep interior structure, or detect problems in the assumed physics. Without such information, parts of the precious seismic data must be used to constrain the overall stellar parameters.

PIERRE MAXTED: I want to mention my experience of CM Draconis, a star that has been mentioned before. This star determines the effective temperature scale for low mass stars, provides tests of models of stellar structure through its accurately known mass, radius and luminosity, and also allows limb darkening to be directly measured. Yet, I only heard of this star one year ago. I think this demonstrates the need for more interaction between observers and theoreticians, so that observers can observe the stars that best constrain the theoreticians' models.

BENGT GUSTAFFSON: A philosophical remark on your philosophical introduction to what is worthwhile and important to concentrate on and what is 'weather.' For a long time we have concentrated on fundamental principles, which I guess is a heritage from physics. This is alright, but there is a growing interest in natural sciences as regards complexity and formation of structure. Now, stellar physics offers a plethora of complex structure, both in atmospheres and, as we begin to understand, in their interiors. This has its own beauty that many of us love, while others get disappointed. However, one should note that stars, as compared to many other systems in nature, are still reasonably simple and admit understanding. They seem, therefore, to offer examples of structure formation that are suitable as case studies in a much wider context. 